\title{
An unusual cause of dysphagia: a vascular ring
}

\author{
R.J. Hall and Tom Treasure \\ Department of Cardiothoracic Surgery, St George's Hospital, Blackshaw Road, Tooting, London \\ $S W 170 Q T, U K$
}

Summary: A 40 year old woman presented with dysphagia which was due to one of the forms of vascular ring. Her symptoms were relieved by relatively conservative surgery. The means of diagnosis and the surgical approach are discussed.

\section{Introduction}

There are a number of well-recognized patterns of congenital anomaly of the great arteries which are of clinical importance when they compress the trachea and/or the oesophagus. The features in this case are a right aortic arch, a retro-oesophageal component, and a fibrous chord representing the atretic or obliterated residue of the left aortic arch. ${ }^{1}$

\section{Case report}

A 40 year old woman was referred by her general practitioner to her local district general hospital physician with a 7 month history of gradual onset, progressive dysphagia. Routine examination showed her to be well nourished without any other notable features. A chest X-ray did not have the usual appearance of a 'knuckle', suggesting absence of the normal left-sided aortic arch (Figure 1). A barium swallow showed posterior indentation of the oesophagus at a level just above the left main bronchus (Figure 2). Subsequently digital subtraction angiography confirmed a right-sided arch and demonstrated that the left subclavian artery arose from an aortic diverticulum (Figure 3). In this case cross-sectional images were obtained with computed tomographic (CT) (Figure 4) and magnetic resonance imaging (MRI) (Figure 5). These confirmed that the diverticulum ran behind the oesophagus. These features are typical of a vascular ring which, in this case, had a posterior diverticulum, a right-sided arch and an atretic portion of the left-sided arch (not imaged) completing the ring. A left posterolateral thoracotomy was performed through the fourth interspace. The diverticulum was dissected from the surrounding structures. The band ran from its apex, anterior to the oesophagus, deep into the

Correspondence: R.J. Hall, M.B., B.S.

Accepted: 3 December 1993 mediastinum. It was divided and, as the ends sprang apart (by about $2 \mathrm{~cm}$ ), the oesophagus bulged forward, freed from compression (Figure 6). No further action was taken and the chest was closed in the hope (and expectation) that the dysphagia had been relieved without the problems which attend major vascular reconstruction in this area, which include the risk of paraplegia. Within 24 hours she was able to eat pork chop and chips, a meal she could not have swallowed previouslyo Subsequently barium swallow and fibreoptie oesophagoscopy confirm that there is no obstruc tion. She remains well a year later.

\section{Discussion}

The first suspicion that a vascular ring was the cause of her dysphagia was the abnormal chest

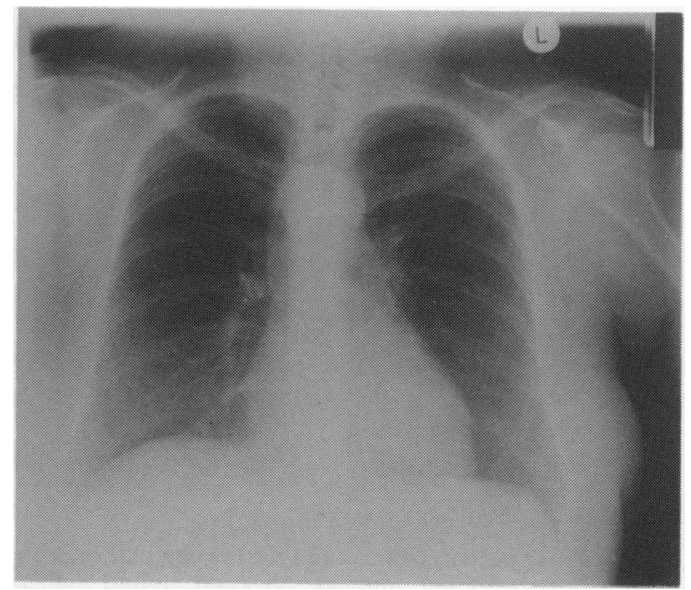

Figure 1 PA chest radiograph with an abnormal appearance of the aortic shadow due to a right-sided aortic arch. 
radiograph (Figure 1) and the barium swallow confirmed that obstruction was extrinsic and at the left of the expected vascular anomaly. This is diagnostic in nearly all cases. ${ }^{2-4}$ However, the majority of examples are in children ${ }^{5}$ where the diagnosis may be made on the basis of pattern recognition because there is no other likely explanation for tracheal and oesophageal obstruction presenting in early life. In a middle-aged woman presenting with dysphagia we asked for more evidence before feeling confident about her diagnosis. After angiography we proceeded to CT scan and MRI in the hope of demonstrating the anatomy of the ring, the exact site of constriction and, ideally, the non-vascular component. Ultim-

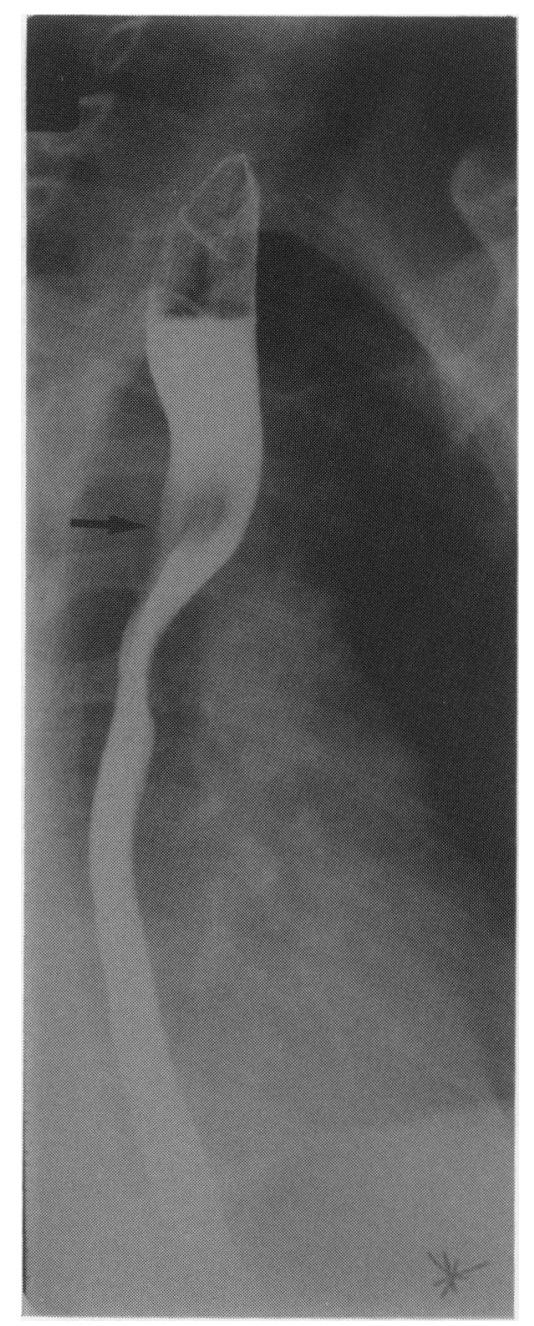

Figure 2 Barium swallow showing posterior indentation of the oesophagus at the level of the aortic diverticulum (arrowed).

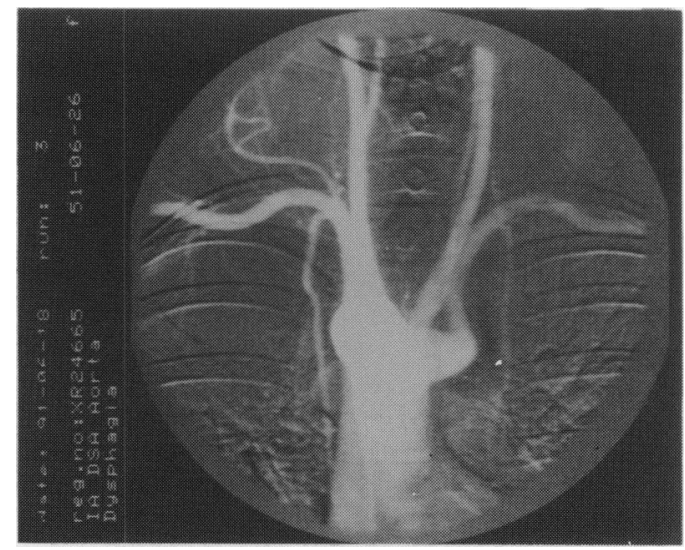

Figure 3 Intra-arterial digital subtraction angiogram of aorta showing a right-sided arch and a left subclavian artery arising from an aortic diverticulum.

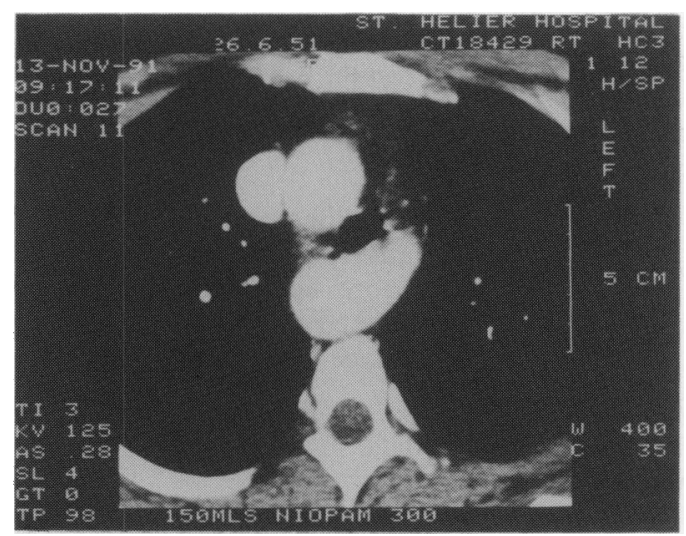

Figure 4 A CT scan (with contrast) just below the level of the aortic arch showing the aortic diverticulum passing from right to left immediately in front of the vertebral body.

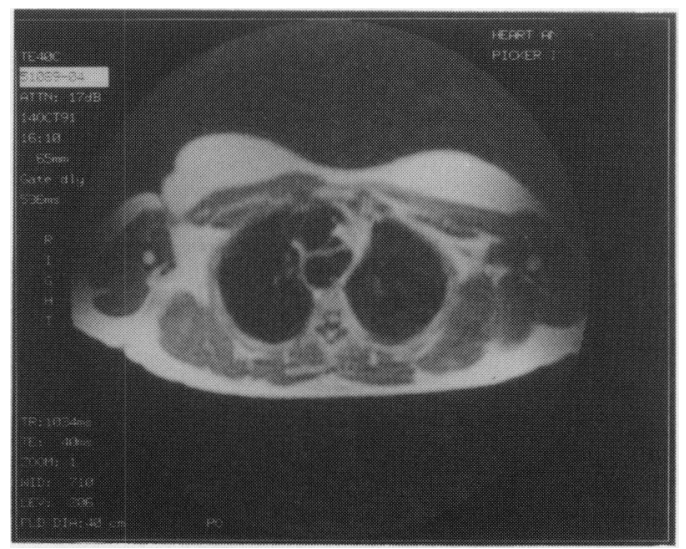

Figure 5 A magnetic resonance image at much the same level as the CT scan. 


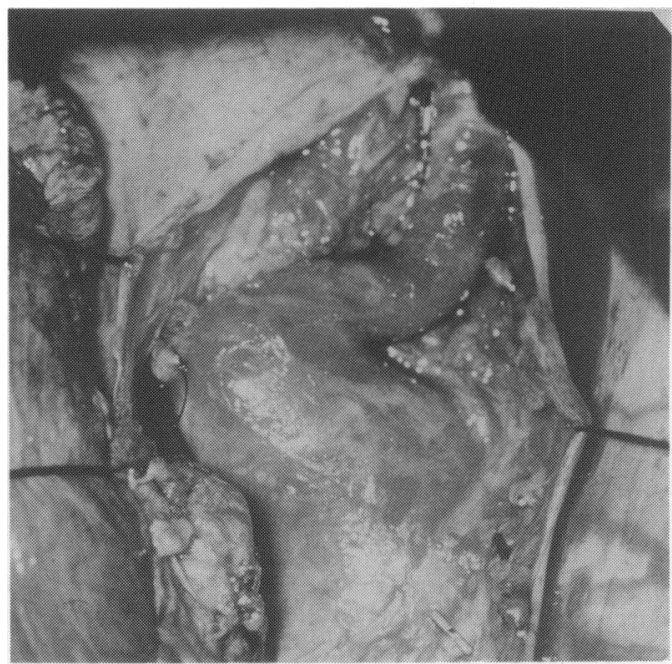

Figure 6 An operative photograph of the diverticulum and the left subclavian artery arising from it. The suture marks the point at which the band was divided.

ately they made little difference, although when reviewed postoperatively, the features of the abnormality were more apparent. It has been suggested that three-dimensional reconstruction of MRI images may replace angiography. ${ }^{6}$

\section{References}

1. Kirklin, J.W. \& Barrat-Boyes, B.G. Vascular rings and slings. In: Cardiac Surgery. John Wiley \& Sons, New York, USA, 1986, pp 1111-1128.

2. Chun, K., Colombani, P., Dudgeon, D. \& Haller, J. Diagnosis and management of congenital vascular rings: a 22 year experience. Ann Thorac Surg 1992, 53: 597-603.

3. Lincoln, J.C.R., Deverall, P.B., Stark, J., Aberdeen, E. \& Waterston, D.J. Vascular anomalies compressing the oesophagus and trachea. Thorax 1969, 24: 295-306.

4. Richardson, J.V., Doty, D.B., Rossi, N.P. \& Ehrenhaft, J.L. Operation for aortic arch anomalies. Ann Thorac Surg 1981, 31: 426-432.
This case illustrates three issues in surgical decision making. The first is that the diagnosis falls $\underset{ }{z}$ outside common experience and is neither the likely $\stackrel{\varnothing}{\varnothing}$ cause of dysphagia in this age group, nor the typical $c$. presentation of this congenital anomaly. Therefore $\overrightarrow{\vec{F}}$ caution and thorough diagnostic information are $\stackrel{9}{9}$ required. The second is that the operative approach is critical. From first principles, it could be argued that a right thoracotomy would give best control of $\frac{\widehat{ }}{\overparen{D}}$ the aorta, or that median sternotomy would give $\varnothing$ best control of the heart and great vessels, but it is $\%$ only through a left thoracotomy that the band, $\vec{O}$ which reference to standard texts ${ }^{7}$ tells us is the actual cause of the problem, can easily be reached. Third, sometimes the best operative solution is parsimonious. At operation the fibrous band was $\frac{0}{7}$ divided leaving the retro-oesophageal vascular $\stackrel{3}{-}$. diverticulum. This was considered to be sufficient $\partial$ to relieve her obstruction without subjecting her to aortic reconstruction with its attendant risk of $-v$ paraplegia, although the diverticulum may be $\stackrel{\$}{\infty}$ resected or retracted by a suture. ${ }^{2}$

One remaining puzzle is why this abnormality should begin to cause trouble after 40 years. It is $\overrightarrow{C P}$ possible that age-related elongation and dilatation $\frac{\mathbb{D}}{O}$ of the arterial system caused obstruction where it $\underset{\mathbb{D}}{\vec{D}}$ had not been constricting before. Alternatively, $\frac{3}{0}$ oesophageal motility may have been adequate to $\underline{\Phi}$ overcome resistance but had then become abnof $\overrightarrow{0}$ mal. We have no evidence to support or refue either theory.

5. Bertolini, A., Pelizza, A., Panizzon, G. et al. Vascular rings and slings. J Cardiovasc Surg 1987, 28: 301-312.

6. Azarow, K.S., Pearl, R.H., Hoffman, M.A., Zurcher, R., Edwards, F.H. \& Cohen, A.J. Vascular rings: does magnetic resonance imaging replace angiography? Ann Thorac Surg 1992, 53: 882-885.

7. Wychulis, A.R., Kincaid, O.W., Weidman, W.H. \& Danielson, G.K. Congenital vascular ring: surgical considerations and 3 results of operation. Mayo Clinic Proc 1971, 46: 182-188. 